\title{
ZU HARTMANNS LYRIK.
}

Naumaun will Beitr. 44, 299 nach dem vorgange von Bech das 212,18 in $\mathrm{BC}$ ïberlieferte wisse als wizze deuten. Ich halte dies für unstatthaft. Man soll dem dichter der kristallenen wörtlein nicht ohne not eine sprachliche uncorrectheit zuschieben, und das wäre der conjunctiv wizze, der in keiner weise syntaktisch gerechtfertigt werden kann. Das praeteritum ist vom standpunkt des futur'ums d $\hat{a}$ wil ich geniezen 'mir wird wohl zugute kommen' aus gesetzt. In der zukunft wird man sagen können: sie hat recht gut gewußt, weshalb ich ihr fern blieb. Ob Hartmann an unserer stelle wesse oder weste gesagt hat, kann niemand wissen. Daß Hartmann wesse nur einmal reimt, erklärt sich durch die schwierigkeit der bindung. Es könnte sogar das überlieferte wisse richtig sein; auch diese form ist nicht leicht zu reimen.

Es ist sehr dankenswert, daß Naumann s. 300 darauf aufmerksam macht, daß zwei lieder, deren echtheit bisher nicht angezweifelt worden ist, abweichungen von Hartmanns sprachgebrauch zeigen. Von diesen liedern nimmt 215,14 anch durch seinen daktylischen rhythmus eine sonderstellung ein. Ich möchte es trotzdem nicht mit sicherheit Hartmann absprechen. Daß Hartmanns sprache nur sante, wante usw. gemäß war, hat Zwierzina überzeugend dargetan; wenn sich aber Hartmann trotzdem in seinen ersten werken erlaubt, sande zu reimen, so könnte er sich immerhin einmal auch ein bewande gestattet haben.

Aber die bedeutung der reime undertân : gewan und fünde : künde: ünde in den liedern 211,27 und 212,37 scheint mir Naumann doch zu unterschätzen. Hartmann reimt in allen seinen werken, vom Büchlein bis zum Iwein, die conjunctive praet. der verba des typus vinden nur auf sicheres unde(n). Und diese bindungen sind recht häufig. ${ }^{1)}$ Andererseits bringt er es im Gregorius fertig, dem wort iinde viermal zu einem partner zu verhelfen. Und da soll er in dem einen lied nicht ohne einen für ihn falschen reim haben auskommen können?

1) Vgl. Kraus, Abb. z. germ. philologie s. $113 \mathrm{f}$. - S.114, z. 21 ist nicht wie Nolte, Zs. fda. 51, $138 \mathrm{anm}$. will, statt 261 die zahl 313 zn setzen, vielmehr ist im druck nach 261 ansgefallen: grumde : kiude (pract); :31:3. 
Fr hat wohl ibberhaupt nicht gewußt, daß es leute gab, die rïude sprachen, sonst würde er es im Iwein vermieden haben, diese conjunctive in den reim zu stellen. Und was den reim -al : $-\hat{i} n$ betrifft, den er freilich bei andern finden konnte, so scheinen mir $Z$ wierzinas ausfihrungen $Z$ s. fda. 44, 363, anm. 2 unwiderleglich. Lachmanns gewaltsame änderung könnte man aber nur billigen, wenn Hartmanns verfasserschaft über jeden zweifel erhaben wäre. Das ist sie aber durchaus nicht.

Das gedicht $211,27-212,12$ ist nach meiner festen überzeugung nicht von Hartmann. $Z u$ seiner art stimmt ganz und gar nicht der spielerische, leichte, ja übermütige ton dieser strophen. Denn übermütig ist der ton; man muß nur den text richtig deuten. Die erste strophe knüpft freilich an einen gemeinplatz an, 1) aber der dichter gibt ihm eine originelle, paradoxe wendung. Daß auf regen sonnenschein folgt, ist banale weisheit; unser dichter zieht aber daraus die kühne folgerung, daß mißgeschick besser ist als glück. Der sinn der ersten strophe ist: wenn es mir schlecht geht, so denke ich mir, es wird schon wieder gut werden, und insofern bin ich besser daran als einer, dem es gut geht. Denn wenn der traurig ist, so hat er nicht einmal den trost, daß ein umschlag des schicksals ihn froh machen werde; der umschlag kann ja nur ein umschlag ins schlechtere sein. ${ }^{2}$ ) Und der erste vers der dritten strophe spricht es klipp und klar aus: ez ist mir iemer mêre guot daz mîn unstaete an stceten fröiden mich versûmet hât. Man sieht, wie wichtig Vogts feststellung ist, daß auch $\mathrm{B}$ ist, nicht wirt schreibt. Der ton des gedichtes weicht

1) Näher als der Spervogelspruch MF. 20, 25, auf den Saran und Bech verweisen, scheint mir die im Reinhart 1028f. citierte äußerung Walters von Horburg zu stehen: ez komet mir als lîhte ze guote, sô ez mir tuot dehein ungemach. - Vgl. übrigens Folquet von Marseille ed. Stroński XIII, 28 ff.: Mas ieu avia plivensa tant quant amei follamen, en aisso qu'om vai dizen: te fenis qui mal comensa. Peire Vidal dreht dies nack seiner weise: $e$ quar anc no fis falhensa, sui en bona sospeisso que $l$ maltraitz mé torn en pro, pos to bes tan gen comensa (Bartsch, Chrestom. provençale 4 108).

2) Hier liegt vielleicht zugleich eine polemik vor gegen die anschauung, die vertreten ist in den Disticha Catouis IV, 26: Tranquillis rebus semper quae adversa timeto. Rursus in adversis melius sperare memento und von Horaz Od. II, 10,13 ff.: Sperat infestis, metuit secundis alteram sortem bene praeparatum pectus. 
also ganz ab von der ernsten, schweren art der Hartmannschen lyrik. Und dazu kommt dann noch der reim undertân:gewan!1) Was den text betrifft, so möchte ich zur erwägung stellen, ob 212, 6 nicht mit B vrouwen statt fröiden $\mathrm{zu}$ lesen ist. Fs liegt hier einer der fälle vor, wo die von mir Beitr. 43, 13 erschlossene doppelbedeutung von vrouwe das auseinandergehen der ubberlieferung bewirkt hat. ${ }^{2}$ ) An unserer stelle ist nun nicht einzusehen, weshalb das keinen ansto $B$ gebende fröiden von einem schreiber geändert worden wäre. Eine zweite frage ist dann, ob der dichter mit vrouwen 'freuden' oder 'frauen' gemeint hat. Ich glaube 'frauen'. Denn für den begriff 'freude' scheint vrouwe nur $\mathrm{zu}$ wortspielerischen zwecken verwendet worden zu sein. Konnte man aber sagen unstote hât mich an vrouwen versûmet? Die im Mhd. wb. 2, 2, 729 b, 26 angeführte stelle aus Berthold läßt in ihrer vereinzelung - auch Lexer bringt keinen anderen beleg - nicht den schluß zu, daß dasjenige, in bezug auf welches die schädigung eintritt, eine sache sein müsse. Vgl. übrigens die im Mhd. wb. 730a, 26 citierte stelle aus Walther (120,24): daz si an mir ouch niht versûme sich. ze statt an MF. 116, 25.

Der plural vrouwen, da doch nur eine gemeint ist. vergleicht sich dem oft vorkommenden plural von friunt für die geliebte. Vgl. MF. 43, 11. 48, 8. 107, 37. 116, 2. 156,15. 181, 37. 214,25; Rubin, Bartsch, Liederdichter 51,51; Vogt zu MF. 116, 2 und 214,25; Schissel von Fleschenberg, Das adjectiv als epitheton im liebeslied des 12. jh.'s, s. 16. Eine interessante parallele gibt die von Singer, $\Lambda$ bh. d. preuß. akad. d. wiss. 1918. phil.-hist. klasse nr. 13, s. 12 citierte bemerkung von Dalman. Palästinischer diwan s. XIII: ' $\mathrm{Es}$ ist eine eigentümlichkeit arabischer liebeslieder, daß der dichter es zuweilen liebt, sogar von freunden in der mehrzahl zu reden, wenn er doch nur eine freundin meint'. Aber die deutschen plurale gehören doch wohl in einen größeren syntaktischen zusammenhang. Vgl. Osthoff, IF. 20,202 ff. Ein mhd. beispiel außerhalb der lyrik ist Nih. 420, 4: (wie fliesen wir dın lîp), suln uns in discm lande mu

1) Den schoren gruoz 211,38 hat Naumamn nicht richtig verstanden. ler simn ist anmutige rele: 'Sie sprach so lielenswiirdig mit mir'.

2) C. v. Kraus hat mich norh auf folgende stelleu nufmerksam gemachit: MF. $15 \%, 14$. $23 ; 159,17 ; 175,29 ; 179,4$. Vgl. anBerdem $: 35$, ! 


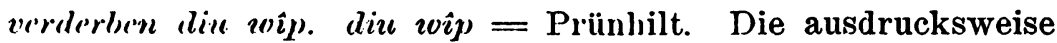
ist dort, am plat\%e, wo es mehr auf den begriff als auf das individuum ankommt.') Iaß sie dann auch zu verhüllenden zwecken angewandt wurde, soll nicht bestritten werden.

Die echtheit des gedichtes 212,37 ist schon, bevor Kraus auf den entscheidenden reim hingewiesen hatte, von Wilmanns und Burdach aus inhaltlichen oder stilistischen gründen angefochten worden. Ich habe dem nichts hinzuzufügen und will nur einiges zum text bemerken. Bechs interpunction, punkt nach 38, komma nach 213,1 und seine übersetzung: 'mich überwand die schlaue list dessen, der mir oft falsch schwört' ist unrichtig. Es sieht dann so aus, als ob die sêle nern und heilic wesen dasselbe wäre. In wahrheit liegt eine steigerung vor: 'wenn man durch lügen seine seele rettet, so ist der meineidige heilig'. Das meine swern übertrifft das liegen ebenso wie das heilic wesen das die sêle nern.

suochte 213,9 ist nicht wie Säran und Bech annahmen, indicativ, sondern conjunctiv: 'warum sollte ich andere um rat fragen, da mich mein eigenes her'z betrogen hat?' Die anknüpfung an das vorhergehende ist auch so schwierig. Es ist wohl der gedanke zu ergänzen: mit meinen klagen will ich nicht etwa rat verlangen; das hätte keinen sinn, denn wie könnten mir fremde helfen, usw. Die begründung ist rein rhetorisch, bloß auf den gegensatz von frömde und mîn sclber aufgebaut. Rat sucht man natürlich nur dann, wenn man sich selbst nicht zu raten weiß. Aus der tatsache, daß man sich selbst schlecht beraten hat, $\mathrm{zu}$ folgern, daß andere noch schlechter raten würden, ist ein sophisma, ermöglicht durch die erfahrung, daß man aus egoismus für sich besser sorgt als für andere. Aber nur dann kann man das tun, wenn man gescheit ist, seine sache versteht, und das war hier eben nicht der fall. Ich glaube, daß dem dichter die worte Reinmars (MF. 170, $36 \mathrm{f}$.) im ohr klangen: Niemen seneder suoche an mich deheinen rât : ich mac mîn selbes leit erwenden niht.

1) Auch heute ist diese ausdrucksweise noch geläufig. 'Das haben Sie auch vor collegen gesagt', hörte ich einmal jemand äußern. Er konnte nur einen collegen meinen; es kam ihm aber darauf an, daß die bemerkung nicht unter vier augen gefallen war. - Die beispiele aus dichtern, die Blatz, Nhd. gramm. ${ }^{3}$ II, 191 bringt, sind nich' gleichartig und beruhen zum teil gewiß auf nachahmung des lateinischen. 
Naumann bemerkt, daß die form friunde 216,8 gegen Hartmanns sonstigen sprachgebrauch verstößt, und fragt, ob hier nur die alternative zwischen einer besserung oder der unechtheit des liedes 216,1 bestehe. Ich lasse es unerörtert, wie ich antworten wïrde, wenn das gedicht nur diesen anstoß böte, und lasse statt dessen eine eingehendere untersuchung des textes folgen.

216,1 Swes fröide hin ze den bluomen stât, der unuoz vil schiere trûren gegen der swæren zît.

gegen fasse ich nicht wie Bech temporal 'um die zeit des herbstes oder winters'. Vielmehr: entgegentrauern, d. h. über die swaere zît trauern. Vgl. Walter 120,13 unde spilet im sîn herze gein der wünneclîchen zît und MF. 117, 19 sô stîgt nî̀n fröide gegen der wunneclîcher zît, ferner Neidhart 85, 10. 86,32. - wirt z. 3 ist futurisch.

8 Die friunde habent mir ein spil geteilet vor, dêst beidenthalp niht wan verlorn:

10 doch ich ir einez nemen wil, âne guote wal sô wære ez baz verborn. si jehent, welle ich minne pflegen, sô müeze ich mich ir bewegen:

doch sô rætet mir der muot ze beiden wegen.

Diese strophe ist voller schwierigkeiten. Zunächst: was heißt z. 11? Wir sind geneigt zu übersetzen: 'wenn man nicht gut wählt, so wäre es besser, das spiel zu lassen'. Aber das mädchen $m u ß$ ja wählen, da die verwandten sie vor ein entweder-oder gestellt haben, und wie sie auch wählt, sie muB verlieren. Einigermaßen käme man zurecht, wenn man erklären dürfte: 'da keine gute wahl möglich ist, wäre es besser das spiel zu unterlassen'. Aber kann der mhd. ausdruck das bedeuten? Eine sehr entfernte parallele gäbe z. 5. 6: sus wil ouch ich den winter lanc mir kürzen âne vogelsanc, insofern es nicht in der macht des mädchens steht, sich den winter über mit vogelgesang zu ergötzen.

Dann der satz doch ich ir einez nemen wil. Man könnte ilhn auch zu dem vorhergehenden ziehen. Aber in jedem fall wäre das zu erwartende swederz ich neme (nome). Die mit. doch eingeleiteten sätze sind nicht immer concessiv; ${ }^{1}$ ) vgl.

1) Die function von doch ist, auf irgendeinen gegensatz hiuzudeuten; das concessive verbältnis ist uur ein specialfall. Richtig iber Otfids 
Hrec 4162. 8911. 9295; Par\%. 406, 10. 742,10; MF. 115,21. Aber mit einem 'ich will 'zvar' oder 'während ich will' kommen wir gar nicht durch. Denn sie will ja nicht, vgl. z. 14. Deshall) wißite auch, wenn doch rein concessiv wäre, der conj. pratet. stehen: 'wenn ich auch eines nehmen wollte'. Es ist kein vernünftiger sinn herauszubringen, und zu conjicieren etwa keinez oder gar devvederz für einez - geht nicht an.

15 Wære ez miner friunde rât, jâ herre, wes solt er mir daune wizzen danc?

sît erz wol gedienet hât, dâ von sô dunket mich sîn bîten alze lanc: wand ich wâgen wil durch in

20 den lîp die êre und al den sin; sô muoz mir gelingen, ob ich sælic bin.

Z. 17 möchte ich mit Bech zum vorhergehenden ziehen. Aber seine auffassung der stelle - Schönbach, Hartmann von Aue s. 369 hat dieselbe - ist unrichtig.

Das mädchen setzt nicht den fall, daß sie nach dem wirklich ausgesprochenen wunsch ihrer verwandten handelt, $d . h$. den geliebten fahren läßt, sondern daß die verwandten ihr zureden, den mann zu nelımen. Wenn sie einfach täte, was ihre verwandten wollen, so hätte sie kein verdienst; wenn die verwandten einverstanden wären, hätte der geliebte keinen grund, ihr dankbar zu sein, während er doch durch seinen dienst anspruch darauf hat, daß sie ihm etwas zuliebe tut. So hat auch der dichter des 2. Büchleins $157 \mathrm{ff}$. die stelle verstanden. ${ }^{1}$ )

Der causalsatz z. 19 gibt keinen vernünftigen sinn. Daß sie für den geliebten alles aufs spiel setzen will, ist kein grund dafür, daß es ihr scheint, daß er schon zu lange warte. Zudem hat z. 18 ihre begründung in z. 17 und weist darauf hin durch das einleitende $d \hat{a}$ von. Ich glaube, wand steht für wan: 'aber ich will für ihn wagen'.

Ja, aber was will sie wagen? den lîp und die êre, das

gebrauch W. Schnatmeyer, Otfrids und seines evangelienbuches persönliche eigenart (Greifswalder diss. 1908), s. 30. Vgl. auch Notker I 299, 25. 396, 1. 800,8. Für die sprache des Heliand: Behaghel, Die syntax des Heliand s. 342 .

1) Vgl. zum ausdruck Walter 97, 1: Wer sol dem des vizzen danc, dem von stcete liep geschiht, nimt der state gerne war? 
kann man wagen, aber den sin? Bech erklärt al den sin durch 'mein ganzes her\%, all mein fühlen und denken'. Kaun man sein her\%, sein fühlen und denken aufs spiel setzen, wie man leben und ehre aufs spiel setzt? Schönbach s. 369 übersetzt 'mein ganzes wesen', was al den sin nun und nimmermehr bedeutet. Übrigens wird auch durch diese falsche übersetzung die schwierigkeit nicht behoben. Sie ist eben einfach nicht zu beheben.

22 Er ist alles des wol wert, ob ich mine triuwe an in behalten wil, des ein man ze wîbe gert:

25 dêswâr dekeiner êren ist im niht ze vil. er ist ein sô bescheiden man, ob ichz an im behalten kan, minne ich in, dâ missegât mir niemer an.

Der $o b$-satz z. 23 ist nicht streng logisch. Die würdigkeit. des mannes ist nicht durch die treue des mädchens bedingt. Gemeint ist: 'wenn ich mich ihm gegenüber treu erzeigen will, muß ich ihm gewähren, was ein mann vom weib verlangt, und dessen ist er würdig'. Aus dieser ineinanderschiebung. zweier gedanken darf man dem dichter keinen vorwurf machen. Aber der zweite ob-satz z. 27! Unmöglich ist Bechs erklärung: 'wenn ich, falls ich ihn liebe, ihm meine liebe $\mathrm{zu}$ bewahren suche, so kann mir daraus kein nachteil erwachsen'. minne ich in gehört unmittelbar zu $d \hat{a}$-an. Läßt man den ob-satz zunächst beiseite, so ergibt sich ein ganz guter sinn: 'er ist ein so verständiger mann, daß mir aus der liebe zu ihn kein schaden erwachsen wird'. Welche syntaktische function hat nun aber der ob-satz? Den satz minne ich in kann er nicht bestimmen; das ergäbe nicht nur dem sinn nach ein monstrum, sondern auch eiuen abscheulichen tonfall. Der ob-satz schließt sich, wenn man ihn richtig vorträgt, genau so wie der ob-satz z. 23 an den vorhergehenden satz an und ist wie ein nachtrag zu sprechen. Aber syntaktisch gehört er nicht zu z. 26. Tielmehr gibt er die voraussetzung für das ganze satzgefüge an. Die bescheidenheit des mannes und ihre folgen können überhaupt nur dann in die erscheinung treten, wenn das mädchen ihm ihre treue erweist, d. h. sich ihm hingibt.

Statt ichz 7. 27, das keinen sinn gibt, ist ichs zu schreiben, si $=$ mine trinwe. Diese beziehung des pronomens übes drei 
verse hinweg ist hart. Aber das gedicht ist überhaupt voller hïrten und anstöße, wie man sie sonst nicht bei Hartmann findet. I) kou kommt ein sprachtechnisches moment. Hartmann setzt in seinen liedern zwar mîn öfters hinter das substantiv, aber in keinem sicheren fall ein attributives adjectiv. Denn tîsentvalt 218, 3 ist adverb wie Iw. 8008 und frowe guot 214, 34 steht in einem unechten lied. In unserem gedicht finden wir aber 216,5 das unschöne den winter lanc.

Ich glaube, daß das lied Hartmann abzusprechen ist.

Bei dieser gelegenheit möchte ich auch zu ein paar anderen gedichten einige bemerkungen vorbringen.

205, 1 Sit ich den sumer truoc riuw unde klagen

sô ist ze fröiden mîn trôst niht sô guot,

mîn sanc ensïle des winters wâpen tragen.

Ich fasse sît nicht wie Bech temporal, sondern begründend.') 'Da ich schon den sommer über traurig war, habe ich jetzt, in der schlechten jahreszeit, keine hoffnung fröhlich zu werden'.

Die wiederholung truoc-tragen ist beabsichtigt. Es liegt eine art wortspiel vor: das wâpen des winters sind riuwe unde klagen.

205, 14 mîn vrowe gert mîn niht: diu schulde ist mîn.

sît sinne machent sældehaften man

und unsin stæte sælde nie gewan,

ob ich mit sinnen niht gedienen kan,

dâ bin ich alterseine schuldec an.

Saran übersetzt Beitr. 23, 16 f.: 'Denn da nur lebensklugheit den mann glücklich macht, torheit aber nie ein dauerhaftes glück erlangt, so bin ich daran, falls ich wirklich nicht mit verstand zu dienen weiß, eben ganz allein schuld'. v. $18 d \hat{a}$ -an gehe auf v. 14.

Aber es ist ganz unnatürlich, v. 18 auf etwas anderes zu beziehen als auf v.17. Dann ist auch die begründung seltsam: weil nur klugheit glück verleiht, ist der unkluge schuld an seinem unglück.

Freilich, wenn man v. 18 auf 17 bezieht, scheinen andere schwierigkeiten zu entstehen, aber nur dann, wenn man sich

1) Die möglichkeit causaler deutung gibt auch zu J. Heymann, Die causalsätze der deutschen lyriker im 12. jh., Berliner diss. 1903, s. 46. 
verleiten läßt, den sît-satz als bestimmung des satzgefüges 17.18 zu nehmen. In wahrheit ist er ein nebensatz zweiter ordnung, eine ergïnzung, und zwar eine freie, von 17 . Wir können ihn zunächst weglassen. 'Die schuld liegt nur an mir', sagt Hartmann 14, und nun die begründung: 'denn niemand anderer' als ich ist schuld, daß ich nicht mit sinnen dienen kann'. Leicht ergänzt sich der zwischengedanke: 'und da ich nicht mit sinnen zu dienen verstehe, will meine herrin nichts von mir wissen'.

Und nun der sît-satz. sît leitet nicht nur sätze ein, die den grund eines anderen satzes, sondern auch solche, die eine tatsache angeben, die geeignet zu sein scheint, dem im übergeordneten satz gesagten eine andere wendung zu geben. Sie enthalten dann einen grund dafür, daß der inhalt des übergeordneten satzes nicht eintreten sollte. Kurzum, sît kann auch concessive bedeutung haben. Dabei verhält sich concessives sît $\mathrm{zu}$ swie wie causales sît $\mathrm{zu}$ wan oder durch daz. sît gibt subjective gründe und gegengründe an, wan und durch daz objective gründe, swie objective gegengründe. Wir drücken den sinn des concessiven sît durch 'da doch', 'während doch' aus. Vgl. Mhd. wb. II, 2, 321 ${ }^{\mathrm{b}}, 47$; Mensing, Untersuchungen über die syntax der concessivsätze, s. 73.

Diese bedeutung hat sît hier. Daß nur sinne glück bringen, sollte uns veranlassen, mit simnen zu handeln. Das geschieht aber im fall des dichters nicht. 'Nur ich selbst bin schuld daran, daß ich nicht mit sinnen $\mathrm{zu}$ dienen verstehe, während doch sinne allein glück bringen'.

206, 19. Bechs übersetzung: 'wer seine freude findet an edeln frauen' scheint mir den sinn von swes fröude an guoten wîben stât nicht ganz genau wiederzugeben. Vielmehr: 'der, dessen freude durch die existenz guter frauen bedingt ist, der ohne sie keine freude hätte'. Vgl. 216, 1.

\section{2 daz ist mîn site und ist mîn rât,} als ez mit triuwen sol.

Bechs erklärung: 'wie es, wenn man es treu meint, nicht anders sein darf' ist unrichtig. als ez miit triuwen sol ist soviel wie als mit triuwen mîn rât sîn sol. "Ich bin verpflichtet, trell zu raten und das tue ich, indem ich rate, edle frauen zu preisen und ihnen zu dienen'. 

207, 1 Ly ist ein klage und nibt ein sanc dî ich der guoten mite eruiuwe miniu leit.

An den gegensatz von kilage und sanc, von dem Bech spricht, ist hier nicht zu denken. Ienn daß diese strophe mit der strophe 206, 29 zusammenhängt, ist sicher und hier sagt ja der dichter: dâ von muoz ich ir klagen mit sange. Gemeint ist vielmehr: das vorliegende lied wird nicht um seiner selbst willen geschickt, es ist nicht als kunstwerk zu würdigen, sondern nur seinem inhalt nach zu betrachten; dieser inhalt ist eine klage. Es ist ein notbehelf, zu dem der dichter greift, weil er keine gelegenheit hat, mit der dame zu sprechen.

210,37. Schönbach, Hartmann von Aue s. $162 \mathrm{f}$. hat mit recht Zingerles deutung des ausdrucks Kristes bluomen auf die wundmale Christi zurückgewiesen. Der plural bluomen erkläre sich, meint er, aus dem öfter vorgekommenen gebrauche der kreuzfahrer, mehr als ein kreuz auf ihr gewand zu heften. Aber dieser brauch wird durch den artikel bei Du Cange 2,637, auf den sich Schönbach beruft, keineswegs bewiesen. Dagegen ergibt sich aus ihm, daß kreuze auf verschiedene kleidungsstücke geheftet wurden; vgl. auch Du Cange 1,664, spalte 2. Man kann also an rock und mantel denken, wenn man nicht dem dichter die freiheit zubilligen will, statt der ich einen hie trage einfach die ich hie trage sagen zu dürfen.

Wieso das kreuz als blume aufgefaßt werden konnte, wird durch Schönbachs bemerkungen nicht erklärt. Man denke vielmehr an die heraldische stilisierung der blumen, z. b. an die königlichen lilien von Frankreich. Da lag es nahe, im kreuzeszeichen eine solche heraldische blume zu sehen. Dazu kommt, daß die kreuze auf den kleidern von verschiedenen farben waren, vgl. Du Cange 2,637, wie die blumen auf der heide, deren buntheit ja öfters hervorgehoben wird.

\section{1, 8 Mich hât diu werlt alsô gewent daz mir der muot sich zeiner mâze nâch ir sent.}

Bech: gewent 'gewöhnt'. Man darf aber nicht übersetzen: 'die welt hat mich gewöhnt, mich wenig nach ihr zu sehnen'. Es ist von der bedeutung von wenen auszugehen, wie sie im Armen Heinrich 334 und Iwein 3322 vorliegt.to Also: 'die welt 
hat mich derartig an sich gewöhnt, mich zutraulich gemacht, daß' usw. Natürlich ironie.

Die sechs strophen des 209, 25 beginnenden tons sind in MF. auf zwei lieder verteilt: I-IV; V. VI. Saran, Beitr. 23, $19 \mathrm{ff}$. will den schnitt anders fülıren: I. III. V und IJ. IV. VI sollen je einen 'strophenkreis' bilden. Seine ausführungen werden jedoch hinfällig durch die tatsache, daß alle sechs strophen durch den reim verknüpft sind. Giske hat $\mathrm{Zs}$. fdph. 18, 247 nur einen teil der bindungen verzeichnet.1) Es kommen folgende vor: Ia, IIb, VIb: muot : guot; Id man : kan, IIIa an:man; If $w \hat{a} t: h a \hat{t}$, IVa hât : gestât, Vf hât : stât, VId stât:hât; IIf, IVc teil : heil; IIIf entsage : trage, V b tage: trage; V d gar : dar, VIf schar: var.

Der reim verknïpft also nicht nur die ungeraden strophen I und III, III und V und die geraden II und IV miteinander, sondern auch die ungerade $V$ mit der geraden VI und erstreckt sich zweimal auf mehrere teils ungerade, teils gerade strophen: I. II. VI; I. IV. V. VI.

Ebenso werden gerade und ungerade strophen durch wortanklänge miteinander verbunden. Die werlt erscheint nicht nur in II. IV. VI, sondern auch in III $(210,11)$, Kristes in V und VI $(210,37.211,18)$, vgl. auch Krist in III $(210,19)$; fröide und sorge werden einander gegenübergestellt in $V$ und VI $(210,35 ; 211,14.19)$. Saran hat freilich Beitr. 23, 20. 24 die zusammenstellung von sorge in V und VI zurückgewiesen. Aber mit unrecht. Es ist weder hier noch dort an eine bestimmte sorge (und fröide) zu denken. Wenn der dichter 210,35 sagt: mî̀n fröide wart nie sorgelôs, so spricht er einfach die erfahrung aus, daß unsere freuden gewöhnlich nicht rein sind, weil sich unangenehme empfindungen eindrängen. Und die sorge von 211,14 brauchen nicht gerade wirtschaftliche nöte zu meinen. Wieviel gründe gibt es nicht, die einen schon von einer gewöhnlichen reise zurückhalten!

Unzweifelhaft bilden also alle sechs strophen zusammen éin lied. Inhaltlich sind sie freilich nur durch das thema 'kreuzfahıt' zusammengehalten. Hier liegt eine schwäche des dichter's vor. Hartmann hat es nicht verstanden, allgemeingültiges und individuelles auszugleichen. $\mathrm{Fr}$ will einer all-

1) Vgl. auch Naumann, Beitr. 44, 295 f. 
gemeinen stimmung ausdruck geben; unter diesem gesichtspunkt lassen sich III. V. VI betrachten. Aber IV bringt mit der erwähnung des todes des herrn etwas rein persönliches herein. Und I und II enthalten lehren und aufforderungen, nicht ausdruck von stimmungen. ${ }^{1}$ )

212, 27 gewinne ich nâch der langen vrömede schœenen gruoz, wie sêre ich daz mit dienste iemer mê besorgen muoz.

Bech bemerkt: 'besorgen, seine sorge darauf richten, darauf bedacht sein (wieder vergelten)'. Aber 'wieder vergelten' ist etwas anderes als 'darauf bedacht sein'. Nur dieses letztere gibt die bedeutung von besorgen richtig wieder. Der scheinbare bedingungssatz bildet die ergänzung von $d a z 28$, er hat die function eines daz-satzes. Vgl. z. b. Wolframs Willehalm 193, 26 sol iemmer vert amîe mînen lîp umbevâhen, daz mac ir iol versmâhen. Also: 'durch dienen werde ich stets danach trachten, daß man mir nach der langen trennung freundlich begegne'.

214, 12 Niemen ist ein sælic man

ze dirre werlte wan der eine

der nie liebes teil gewan

und ouch dar nâch gedenket kleine.

Bech: 'dar nâch gedenken darauf denken, darnach streben'. Bei dieser auslegung käme ein riß in den gedanken der strophe. Dieser ist einzig und allein: 'niemand ist glücklich als der, dem niemals (liebes)glück widerfahren ist'. Das gedenket kann nicht auf die zukunft, es muß auf die vergangenheit gehen. dar nâch gedenken ist hier soviel wie dar an gedenken.

Bech nahm wahrscheinlich an, daß ouch hier ein neues, coordiniertes glied anfüge. Aber ouch kann auch ein glied anfügen, dessen inhalt in dem vorhergehenden wurzelt, so daß (und) ouch soviel wie unser 'daher' bedeuten kann.') Vgl.

1) Auch die sechs strophen des tones 207,11, die Saran, Beitr. 23, $8 \mathrm{ff}$. auf zwei lieder verteilen will, sind durch den reim auf das innigste aneinandergekettet. Ich glaube, bei keinem lyriker hat man soviel über die zusammengehörigkeit von strophen gestritten wie bei Hartmann; die schuld liegt am dichter.

2) J. Heymann, Die causalsätze der deutschen lyriker im 12.jh., s. 53 bringt nur vier beispiele für causales ouch. Von diesen sind MF. 82, 1 und 73, $7 \mathrm{zu}$ streichen; die durch ouch eingeleiteten sätze stehen zu den vorhergehenden im verhältnis des gegensatzes. 
'Tristan $440 \mathrm{ff} .:$ sus begunden sî sich under in slahen unde morden starke und befulhen ouch dô Marke sich und daz lant in sîne plege.

Hartmanns verse sind vielleicht die umwandlung eines berühmten ausspruchs des Boethius, De consolatione philosophiae II, 4:1) nec inficiari possum prosperitatis meae velocissimum cursum. Sed hoc est, quod recolentem vehementius coquit; nam in omni adversitate fortunae infelicissimum genus est infortunii fuisse felicem. Dem recolrnten würde dar nâch gedenket entsprechen. Der verfasser des 2. Büchleins hat es dagegen (v. 128) ebenso mißverstanden wie Bech und verrät sich auch dadurch als nachahmer.

Einen ähnlichen gedanken wie Hartmanns strophe enthält der anfang des 15. Spervogelischen spruches, Müllenhoff, Altdeutsche sprachproben s. 110, ferner Minnelehre $2127 \mathrm{ff}$.

WIEN.

M. H. JELLINEK.

\section{ZU DEN PSEUDOREINMARISCHEN GEDICH'TEN.}

C. v. Kraus hat in seiner schrift Die lieder Reimars des alten, I. teil (Abh. d. bayr. akad. d. wiss., phil.-philol. u. hist. kl. XXX, 4) eine stattliche anzahl vou liedern Reinmar abgesprochen. Wie ich glaube, in beinahe allen fällen mit recht. Eine wichtige rolle spielte dabei der nachweis der minderwertigkeit vieler dieser gedichte in gedankenführung und sprachlichem ausdruck. Kraus war für seine zwecke durchaus berechtigt, an die lieder den maßstab Reinmarischer formvollendung anzulegen. Aber absolut genommen ist dieser maßstab mitunter zu streng. Ich möchte mich hier zweier durch Kraus namenlos gewordener gedichte annehmen.

1) Er liegt bekanntlich Dantes Nessun maggior dolore che ricordarsi del tempo felice nella miscria (Inferno V,121) zugrunde. - Boethius hat übrigens vorgänger, z. b. Euripides, Iphigenia in Tauris $1117 \mathrm{fr}$. 